\title{
Presentación
}

\section{Los distintos modos de lo urbano}

Al momento de escribir la convocatoria para este número de $C S$, nuestro objetivo fue recoger un registro plural de los distintos desafíos que enfrentan las ciudades latinoamericanas. El número que ahora presentamos reúne de manera representativa algunos de estos y sin duda su lectura posibilita una mirada amplia sobre algunos de los problemas inherentes -recuperando una vieja pero acertada noción- al modo de vida urbano en Latinoamérica. Se incluye, además, un trabajo sobre la relación entre la ciudad y lo rural, espacio que presenta también una acelerada transformación. Adaptando una advertencia del historiador británico James Dyos: es indudable que cada ciudad en cada momento debe probar cuáles son sus límites con el mundo rural que la rodea.

Evidentemente, no se trata de un número convencional sobre la ciudad ni de lo que recientemente se considera como Estudios urbanos. En estos se privilegia la condición urbana como elemento explicativo de las dinámicas sociales que se estudian y que existen justamente por su condición citadina. Los artículos aquí reunidos abordan estas dinámicas de modo muy diferente, algunos se centran específicamente en procesos característicos de las ciudades, teniendo lo urbano como centro de su estructura metodológica y analítica; en otros, lo urbano es un agente, a veces central, a veces periférico, pero siempre insoslayable del juego de relaciones que la ciudad genera.

Esta diversidad refleja bastante bien una conocida tensión entre los trabajos que abordan lo urbano como foco de su interés. Generalmente, se consideran propios del campo académico sobre lo urbano aquellos que lo asumen como el eje central de la explicación que pretenden dar; y, por fuera de él, a aquellos trabajos en los que lo urbano es solo el escenario en el cual los agentes desarrollan su acción. Esta tensión se replica en todo tipo de producciones académicas, sean abordadas disciplinar o interdisciplinarmente, es decir, todas aquellas que pertenecen a campos o subdisciplinas reconocidos en distintas tradiciones académicas. Caben desde el urbanismo, la planeación urbana, la sociología urbana, la antropología urbana, la historia urbana, y una larga lista que incluye el diseño de políticas públicas pensadas específicamente para la ciudad, diversas propuestas estéticas inscritas en la ciudad, e inclusive los llamados Estudios urbanos. Estos últimos son aún difíciles de definir o de agrupar bajo características únicas y claramente definidas; y quizá porque surgieron al calor de los cambios ocurridos en las 
ciencias sociales en la década del 6o, en el marco de la heterogénea producción que se cobijó bajo la sombrilla de los estudios culturales, es que mantienen una condición heterogénea como principal característica.

No es nuestro propósito en estas líneas tomar partido por uno u otro extremo de la tensión, ni siquiera esbozar un conjunto de matices como procedimiento para salir al paso a las dificultades de definición señaladas. Se trata más bien de poner de presente que esta tensión no está resuelta y que quizá sea una de las tensiones constitutivas que explican tanto la condición de lo urbano como la de sus estudios.

La convocatoria trató de recoger de manera amplia artículos que dieran cuenta de lo urbano, y tuvimos como respuesta que los investigadores entendían por lo urbano una diversidad de significados, desde mero decorado hasta centro indiscutible de la experiencia humana. La selección que recoge este número no solo presenta aquellos textos que cumplieron con los estándares de la revista, sino que avanzan en mostrar que la complejidad de lo que ocurre en la ciudad y la complejidad de lo urbano resultan en muchas ocasiones indistinguibles, y que estamos en mora de seguir avanzando en nuevos modos de encarar la tensión antes enunciada, si deseamos que las ciencias sociales no solo presenten y propongan alternativas a problemas concretos, sino que ofrezcan perspectivas integradoras y respuestas equiparables en complejidad a los desafíos urbanos en los que vivimos.

El número presenta, entonces, los resultados de indagaciones diversas que ingresan a la ciudad desde lugares diferentes y llaman la atención sobre procesos distintos. El primer artículo, de Ramiro Segura, da cuenta de la relación entre los patrones de urbanización en América Latina, que en casi todo el continente se caracterizan por ser excluyentes, y las políticas redistributivas que buscan mitigar la abrumadora desigualdad de ingresos en la región. Este trabajo se inscribe en una tradición de estudios sobre los usos del suelo urbano que sistemáticamente han mostrado que la fragmentación urbana no solo es asunto de políticas públicas sobre las formas de urbanización, sino que debe incluir de manera perentoria una consideración sobre las políticas redistributivas de la riqueza, que posibiliten ciudades con una mejor calidad de vida para sus habitantes, y que estos puedan disfrutar de forma más equitativa de las ventajas de la vida urbana.

El segundo artículo también aborda una cuestión clásica de los estudios urbanos, pero no por ello menos urgente: la movilidad. El trabajo de Diana Vinasco Martínez pone en perspectiva uno de los problemas más acuciantes de las ciudades fragmentadas de que nos hablaba Segura en el artículo anterior. La perspectiva histórica asumida por la autora muestra las dificultades y las debilidades de la planeación estatal, a nivel local y nacional, ante el problema de la movilidad urbana; en especial al hacer evidente las dificultades de integrar las zonas periféricas de la ciudad mediante un sistema de transporte que responda al acelerado crecimiento económico de las urbes latinoamericanas a lo largo del siglo. Aunque el trabajo se centra exclusivamente en la ciudad de Cali, en Colombia, 
sus descripciones se asemejan a muchas de las circunstancias de otras ciudades que no son capitales nacionales en América Latina. Estos dos trabajos, por modos distintos, llegan a subrayar los retos de las ciudades en términos de equidad e integración urbanas.

Con el tercer artículo se produce un primer desplazamiento. De los más tradicionales temas urbanos ya presentados, se pasa a otro no menos significativo para la ciudad, pero que la interpela desde sus márgenes, en este caso no a través de formas de exclusión ocurridas en la misma, sino de las relaciones que mantiene con su entorno. El artículo de Natalia Pérez muestra que en ciudades como Cali, como en otras ciudades colombianas, los planes de ordenamiento se centran en los centros poblados y establecen normas que subordinan las zonas aledañas a las urgencias urbanas. La cuenca del río Pance, vecina a la ciudad y lugar tradicional de recreación de la población urbana, ofrece el desafío de considerar su desarrollo no como apéndice de la ciudad, sino como una zona interconectada a ella pero con su propia dinámica, que demanda atención específica, y en la cual el territorio expresa no solo un uso del suelo, sino una serie de desarrollos culturales y productivos que son centrales para conservar la ciudad misma y que son consustanciales a su desarrollo. De central importancia para la valoración de este artículo es el papel de la organización social, que se moviliza en una doble condición, por un lado como pobladores rurales, pero que por el otro mezclan en su accionar y en sus lógicas de relación formas urbanas de entender y dimensionar su vida cotidiana.

Los dos últimos artículos de este número suponen un segundo y último desplazamiento. En ambos casos la ciudad es un factor entre varios que hace posible entender y dimensionar los procesos estudiados. En el caso del trabajo de Dina Alves, la ciudad se desdibuja y aparece como telón de fondo para problemáticas que si bien pueden ser consideradas específicamente urbanas no es bajo esa luz que se las examina, sino como lugar en el cual los procesos de dominación racial sobre mujeres negras en prisión aumentan la vulnerabilidad de esta población. La criminalidad, la violencia y la exclusión de Sao Pablo sirven de contexto para encuadrar estas formas de exclusión.

El artículo de Mateo Pazos Cárdenas aborda el tema de los festivales urbanos mediante una comparación entre el Festival «Petronio Álvarez» en Cali, dedicado a la música del Pacífico colombiano creada por población afrodescendiente, y el «Afrocaribeño» que se lleva a cabo en la ciudad de Veracruz, México. Pazos muestra las dificultades que enfrentan estos proyectos para efectivamente reconocer, al menos de manera institucional, las diversas formas de otredad -para respetar la terminología del autor-. Más allá de las intenciones que los impulsan, estos festivales logran resultados contradictorios con sus propósitos y alcanzan de manera parcial sus pretensiones de reconocimiento de la diversidad multicultural. Estos festivales son característicamente urbanos, aunque las actividades que en ellos se realizan son de origen rural, cada vez están más entroncadas con lo urbano. Aunque este elemento no es central en el análisis de Pazos, su existencia y continuidad requieren del contexto urbano para desarrollarse. 
Cierran estos artículos un documento sobre la conformación de la Red Internacional de Investigadores sobre Encerramientos Residenciales (RIIER). El propósito de esta red es estrechar vínculos entre investigadores e instituciones académicas de todo el mundo para promover la reflexión, el debate, la producción e intercambio de conocimiento a través de publicaciones, encuentros, seminarios y foros alrededor de la temática de los encerramientos residenciales urbanos.

¿Todo lo que ocurre en la ciudad es urbano? Esta pregunta puede surgir después de la presentación de los anteriores trabajos. Seguramente no: las relaciones con el espacio pueden estar condicionadas por el lugar, pero hay también suficiente evidencia para mostrar que en lo urbano perviven formas deslocalizadas que más que rurales, representan otros formas de relación que escapan a lo urbano. Sin embargo, la ubicuidad de lo urbano crece en la medida en que no está contenido en los límites de la ciudad. La presencia en las zonas rurales de condominios y otros tipos de viviendas y parques de recreo no convierte a sus pobladores y visitantes en campesinos, pues las relaciones que allí se proponen son tan urbanas como las del centro de la ciudad. Es evidente que cada caso demanda definir específicamente hasta dónde se proyecta lo urbano y hasta dónde las nuevas ruralidades no deben confundirse con manifestaciones de la vida en ciudad. Aunque el escenario privilegiado de la modernización ha sido la ciudad, la modernización del campo con sus propias lógicas demanda una atención que debe escapar al urbanocentrismo.

En este número, hemos tratado de mostrar las complejidades de lo urbano ofreciendo una muestra de las distintas maneras en las que la ciudad puede ser considerada en los procesos centrales de las ciudades latinoamericanas. Esperamos que este conjunto de trabajos sea una invitación para que el lector encuentre sus propias conclusiones acerca de los derroteros de lo urbano en América Latina a comienzos del siglo XXI.

\section{Enrique Rodríguez Caporalli}

Abril, 2017 